\title{
EZH2 Modulates the DNA Methylome and Controls T Cell Adhesion Through Junctional Adhesion Molecule A in Lupus Patients
}

\author{
Pei-Suen Tsou, Patrick Coit, Nathan C. Kilian, and Amr H. Sawalha
}

Objective. $\mathrm{EZH} 2$ is an epigenetic regulator that mediates H3K27 trimethylation (H3K27me3) and modulates DNA methylation. The aim of this study was to characterize the role of $\mathrm{EZH} 2$ in $\mathrm{CD} 4+\mathrm{T}$ cells in the pathogenesis of systemic lupus erythematosus.

Methods. EZH2 expression levels were determined in CD4+ $\mathbf{T}$ cells isolated from lupus patients and healthy controls. The epigenetic effects of $\mathrm{EZH} 2$ overexpression in CD4+ $\mathbf{T}$ cells were evaluated using a genomewide DNA methylation approach. Gene expression profiles and microRNAs (miRNAs) were assessed by quantitative polymerase chain reaction, while protein expression was examined by Western blotting. A cell adhesion assay was used to assess adhesion of CD4+ $\mathrm{T}$ cells to human microvascular endothelial cells.

Results. EZH2 and H3K27me3 levels were increased in CD4+ T cells from lupus patients compared to healthy controls. $\mathrm{T}$ cell production of $\mathrm{EZH} 2$ was downregulated in the presence of $\mathrm{miR}-26 \mathrm{a}$ and $\mathrm{miR}-101$, and levels of both miRNAs were reduced in lupus CD4+ T cells. Overexpression of $\mathrm{EZH} 2$ induced in $\mathrm{CD} 4+\mathrm{T}$ cells resulted in significant DNA methylation changes. Genes involved in leukocyte adhesion and migration, including F11R (which encodes junctional adhesion molecule A [JAM-A]), became hypomethylated in CD4+ T cells when $\mathrm{EZH} 2$ was overexpressed. Overexpression of $\mathrm{EZH} 2$ resulted in increases in JAM-A expression and CD4+ T cell adhesion. Preincubation of $\mathrm{EZH} 2$-transfected CD4+ T cells with neutralizing antibodies against JAM-A significantly blunted cell adhesion. Similarly, CD4+ T cells

Supported by the NIH (National Institute of Allergy and Infectious Diseases grant R01-AI-097134 and National Institute of Arthritis and Musculoskeletal and Skin Diseases grant T32-AR-007080).

Pei-Suen Tsou, PhD, Patrick Coit, MPH, Nathan C. Kilian, BSc, Amr H. Sawalha, MD: University of Michigan, Ann Arbor.

Address correspondence to Amr H. Sawalha, MD, Division of Rheumatology, University of Michigan, 1150 West Medical Center Drive, 5520 MSRB1, SPC 5680, Ann Arbor, MI 48109. E-mail: asawalha@umich.edu.

Submitted for publication May 26, 2017; accepted in revised form September 26, 2017. from lupus patients overexpressed JAM-A and adhered significantly more to endothelial cells than to $\mathrm{T}$ cells from healthy controls. Blocking JAM-A or EZH2 significantly reduced the capacity of lupus CD4+ $\mathrm{T}$ cells to adhere to endothelial cells.

Conclusion. The results of this study identify a novel role of $\mathrm{EZH} 2$ in $\mathrm{T}$ cell adhesion mediated by epigenetic remodeling and up-regulation of JAM-A. Blockade of EZH2 or JAM-A might have therapeutic potential by acting to reduce $T$ cell adhesion, migration, and extravasation in patients with lupus.

Systemic lupus erythematosus (SLE) is a chronic, relapsing autoimmune disease that is characterized by autoantibody production and a wide variety of clinical manifestations along with multiple organ involvement. Although the underlying cause of lupus is largely unknown, substantial research has pointed to the involvement of both genetic and epigenetic mechanisms $(1,2)$. In particular, DNA methylation, an epigenetic marker that is heritable, stable, and reversible, has been shown to play crucial roles in mediating the pathogenesis of lupus $(3,4)$. It not only affects gene expression patterns and the function of genes in lupus CD4+ T cells $(5,6)$, but also primes interferon-regulated genes in lupus-naive CD4+ T cells to be epigenetically poised to respond to interferon upon activation (4). CD4+ T cells from lupus patients with active disease show more pronounced defects in DNA methylation as compared to those from lupus patients with inactive disease (7-9).

In a recent study by our group, the findings suggested that with lupus flares, naive CD4+ T cells undergo a shift in DNA methylation to favor $\mathrm{T}$ cell activation. Furthermore, this shift poises CD4+ T cells for Th2, Th17, and follicular helper T cell responses (10). Bioinformatics and literature-mining analyses have demonstrated a significant enrichment of EZH2 binding sites in loci that exhibit progressive increases in DNA methylation levels in conjunction with greater lupus disease activity, 
suggesting that EZH2 has a role in the progression of lupus. In addition, levels of 2 microRNAs (miRNAs) that target and regulate EZH2, miR-101 and miR-26a, in naive CD4+ T cells from lupus patients were found to be negatively correlated with the extent of lupus disease activity.

In this study, we set forth to further evaluate the role of EZH2 in CD4+ T cells from patients with SLE. We sought to determine the expression of EZH2, along with the 2 miRNAs that control EZH2 expression, in naive CD4+ T cells from lupus patients in comparison to healthy controls. Through examination of the changes occurring in the genome-wide DNA methylation status after overexpression of $E Z H 2$ in naive CD4+ T cells, we discovered a novel functional role of $\mathrm{EZH} 2$ in CD4+ $\mathrm{T}$ cells that has potential therapeutic implications in the management of lupus.

\section{PATIENTS AND METHODS}

Patients and controls. All patients included in our study met the American College of Rheumatology revised criteria for the classification of SLE (11). Patients ( $n=57$; all female, median age 42 years [range 19-60 years], mean \pm SD age $40 \pm 11$ years $)$ and healthy controls $(n=40$; all female, median age 44 years [range $20-63$ years], mean \pm SD age $44 \pm 11$ years) were recruited from the University of Michigan Rheumatology Clinics. The SLE Disease Activity Index (SLEDAI) scores (12) in the patients ranged from 0 to 12 , with a mean score of 3 and median score of 2 . All subjects included in this study provided written informed consent. All procedures in this study were reviewed and approved by the Institutional Review Board of the University of Michigan.

Cell isolation and culture. Naive CD4+ T cells were isolated from the whole blood of subjects using a naive CD4+ T cell isolation kit (Miltenyi Biotec), which allows for the indirect isolation of untouched naive CD4+ T cells, as previously described (10). In some experiments, isolated naive CD4+ $T$ cells were left unstimulated or were stimulated overnight with anti-CD3 antibodies $(10 \mu \mathrm{g} / \mathrm{ml}$, precoated on plates) and anti-CD28 antibodies $(2.5 \mu \mathrm{g} / \mathrm{ml})$. Human microvascular endothelial cells (HMVECs) were grown in endothelial basal medium 2 (EBM-2) with growth factors (Lonza). Cells between passages 3 and 9 were used in the experiments.

Overexpression experiments. Overexpression of EZH2 in naive $\mathrm{CD} 4+\mathrm{T}$ cells was performed using an Amaxa 4D-Nucleofactor system (Lonza). After isolation and purification, naive CD4+ T cells from healthy subjects were transfected with $0.1 \mu \mathrm{g}$ of $E Z H 2$, or pCMV6-XL5 as a control vector (both from OriGene), and cultured in RPMI medium supplemented with $10 \%$ fetal bovine serum (FBS) and $2 \mathrm{mM}$ L-glutamine. After 5 hours of transfection, the culture medium was changed to remove the transfection reagent, and the cells were stimulated overnight with anti-CD3 and anti-CD28 antibodies. The cells were cultured for an additional 48 hours before protein and RNA were collected. In addition, DNA was extracted for the DNA methylation assessment (as described below).

Similar procedures were carried out for the miRNA overexpression experiments, using the Amaxa 4D-Nucleofactor system. Naive CD4+ T cells from healthy subjects were transfected with $500 \mathrm{n} M$ of miR-26a or $500 \mathrm{n} M$ of miR-101 (mirVana miRNA mimic; Thermo Fisher Scientific) and stimulated overnight. RNA was collected on day 3 posttransfection. The cell survival rate for the miRNA-transfected cells was $\sim 55 \%$.

Extraction of messenger RNA (mRNA) and quantitative reverse transcription-polymerase chain reaction (qRTPCR). Total RNA from the cells was isolated using a Direct-zol RNA MiniPrep kit (Zymo Research). Preparation of complementary DNA (cDNA) was done using a Verso cDNA synthesis kit (Thermo Fisher Scientific). Primers for human $E Z H 2$, the DNA methyltransferase genes DNMT1 and DNMT3A, the methyl-CpG binding protein gene $M e C P 2$, and $\beta$-actin, along with Power SYBR Green PCR Master Mix (Applied Biosystems), were used for qRT-PCR, which was run using a ViiA 7 realtime PCR system. Primer sequences are as follows: for DNMT1, forward CGACTACATCAAAGGCAGCAACCTG and reverse TGGAGTGGACTTGTGGGTGTTCTC; for DNMT3A, forward CGAGTCCAACCCTGTGATGATTG and reverse CGTGGTCTTTGCCCTGCTTTATG; for $\beta$-actin, forward GTCAGGCAGCTCGTAGCTCT and reverse GCCATGTACGTTGCTATCCA. The $E Z H 2$ primers were KiCqStart SYBR Green primers (Sigma), and the $M e C P 2$ primers were purchased from Qiagen (QuantiTect Primer Assays). Expression of miRNAs was analyzed using TaqMan Advanced miRNA assays (Thermo Fisher Scientific).

Western blotting. Cell lysates were prepared from CD4+ T cells obtained from lupus patients and healthy subjects. Proteins were separated by sodium dodecyl sulfate-polyacrylamide gel electrophoresis, and then electroblotted onto nitrocellulose membranes. EZH2, junctional adhesion molecule A (JAM-A), and trimethylated H3K27 (H3K27me3) were detected using anti-human EZH2 antibodies (Cell Signaling Technology), anti-JAM-A antibodies (Santa Cruz Biotechnology), and anti-H3K27me3 antibodies (Cell Signaling), while $\beta$ actin and histone $\mathrm{H} 3$ were used as loading controls (anti- $\beta$-actin antibodies from Sigma-Aldrich; anti-H3 antibodies from Cell Signaling Technology). Images were visualized using an Omega Lum C imaging system (Gel Company), and quantification of the bands was performed using GelQuant.NET (BiochemLab Solutions).

DNA methylation assessment and analysis. Naive CD4+ T cells from 4 healthy subjects, left unstimulated or stimulated overnight, were transfected with an EZH2 overexpression vector or a control vector. Genomic DNA was then isolated from the cells and bisulfite converted using an EZ DNA Methylation kit (Zymo Research). The genome-wide DNA methylation status of each sample was evaluated using an Illumina Infinium Methylation EPIC BeadChip array. An Illumina GenomeStudio platform was used to analyze the methylation data, as previously described (4). The average level of DNA methylation $(\beta)$ on each $\mathrm{CpG}$ site was compared between the control and EZH2-overexpressing CD4+ T cells. Differentially methylated $\mathrm{CpG}$ sites were defined as those with a differential methylation score of $\geq|22|$ (equivalent to a $P$ value of less than 0.05 , after adjustment for multiple testing) and a mean methylation difference of $>10 \%$ between the 2 groups. Differentially methylated genes were analyzed for Gene Ontology (GO), network, and pathway enrichments using the DAVID Bioinformatics Database (version 6.7) $(13,14)$.

In vitro cell adhesion assay. An adhesion assay to assess the capacity of CD4+ T cells to adhere to HMVECs was carried out as previously described (15), with slight modification. 
HMVECs were grown in 96-well plates and stimulated with tumor necrosis factor $(25 \mathrm{ng} / \mathrm{ml})$ in EBM-2 medium containing $0.1 \%$ FBS. Overexpression of $E Z H 2$ was induced in naive CD4+ T cells in the same manner as described above. The stimulated EZH2-overexpressing CD4+ T cells $(50,000$ cells/well in $100 \mu \mathrm{l}$ RPMI medium) were labeled with calcein AM cell-permeant dye ( $5 \mu M$; Invitrogen), in the presence or absence of neutralizing antibodies to JAM-A (50 $\mu \mathrm{g} / \mathrm{ml}$; BioLegend) or an isotype control (mouse IgG1к; BioLegend). The fluorescence-tagged T cells were washed with phosphate buffered saline (PBS) twice, and then added to HMVEC cultures and incubated for 30 minutes. Nonadherent T cells were washed off, and the fluorescence intensity was measured at an absorbance of 485/528 nm using a Synergy HT fluorescence plate reader (BioTek Instruments).

We also examined the capacity of lupus CD4+ T cells to adhere to HMVECs. Naive CD4 $+\mathrm{T}$ cells were isolated from lupus patients and stimulated overnight. The cells were cultured for an additional 2 days before they were assayed for adhesion capacity in the same manner as described above. The effect of JAM-A in lupus CD4+ T cells was also evaluated using neutralizing antibodies to JAM-A. After culturing for 3 days, stimulated $\mathrm{CD} 4+\mathrm{T}$ cells from lupus patients were washed in PBS and labeled with calcein AM, in the presence or absence of neutralizing antibodies to JAM-A or isotype control. After washing with PBS, the fluorescence-tagged T cells were subjected to adhesion assays, as described above.

To determine whether inhibition of EZH2 would affect $\mathrm{T}$ cell adhesion, naive CD4+ $\mathrm{T}$ cells from lupus patients were stimulated overnight in the presence of DZNep $(5 \mu M$, dissolved in PBS; Cayman Chemical), and 1 day thereafter, the medium was changed to remove the stimulants. The T cells were treated with DZNep for an additional 2 days, and the cells were washed with PBS twice and then assayed for adhesion. The cell survival rate of the DZNep-treated cells was $\sim 77 \%$.

Meanwhile, an aliquot of cells was lysed and saved for Western blotting to check the expression of EZH2, JAM-A, and H3K27me3 after DZNep treatment. To visualize T cell adhesion to HMVECs, dye-tagged stimulated CD4+ T cells from healthy controls, stimulated CD4+ $\mathrm{T}$ cells from lupus patients, and DZNep-treated CD4+ T cells from lupus patients were plated on HMVECs that were labeled with the red fluorescence dye PKH26 (Sigma). Images of the cells were obtained using an EVOS FL cell imaging system (Thermo Fisher Scientific). In order to evaluate the effect of $\mathrm{EZH} 2$ on cell adhesion,

B

A
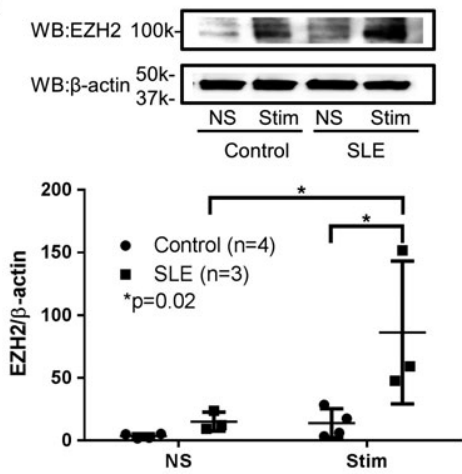

C

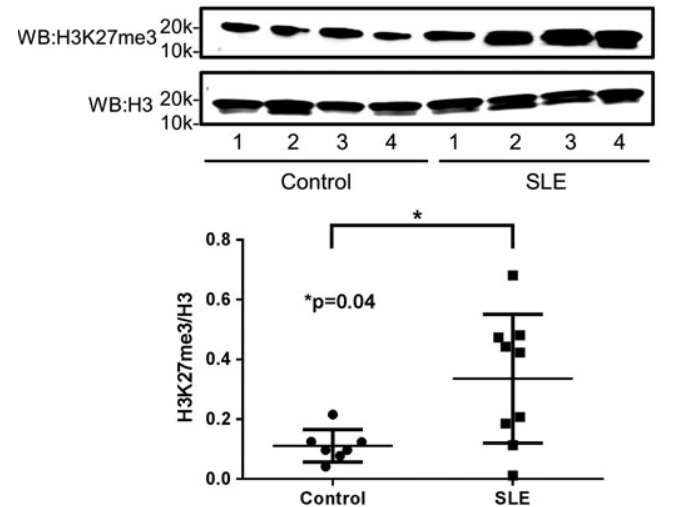

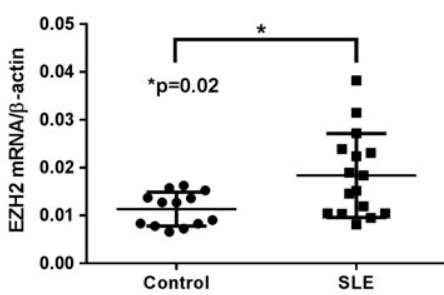

D

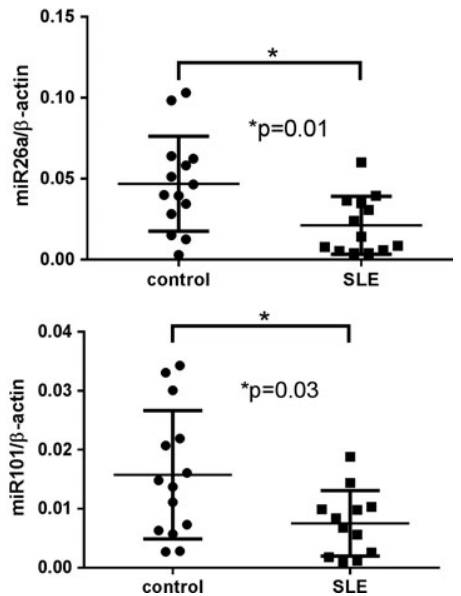

Figure 1. Expression of EZH2, trimethylated H3K27 (H3K27me3), and microRNAs (miRNAs) in CD4+ T cells isolated from healthy controls and patients with systemic lupus erythematosus (SLE). A, Protein levels of EZH2 were assessed by Western blotting (WB) (top) and quantified (bottom) relative to the expression of $\beta$-actin in naive CD4+ T cells from lupus patients compared to healthy controls, with no stimulation (NS) or after overnight stimulation (Stim) with anti-CD3 and anti-CD28 antibodies. B, Expression of EZH2 mRNA was determined in naive CD4+ T cells from lupus patients compared to healthy controls, after overnight stimulation. C, Levels of H3K27me3 were assessed by Western blotting (top) and quantified (bottom) relative to the expression of histone $\mathrm{H} 3$ in naive CD4+ T cells from lupus patients compared to healthy controls, after overnight stimulation. D and E, Levels of miR-26a (D) and miR-101 (E) were determined in lupus and healthy control naive CD4+ T cells, after overnight stimulation. Bars show the mean $\pm \mathrm{SD}$ in each group. 
experiments with GSK126, which inhibits the catalytic activity of EZH2, but not the expression of EZH2 (16), were performed. The procedures used were similar to those used in the experiments with DZNep. The final concentration of GSK126 (Cayman Chemical) was $5 \mu M$, and DMSO was used as the vehicle control.

Statistical analysis. Results are expressed as the mean \pm SD. To determine differences between groups, the MannWhitney $U$ test was performed, and data were analyzed using GraphPad Prism (version 6; GraphPad Software). One-way analysis of variance (ANOVA) followed by Sidak's post hoc test was used to compare differences in EZH2-overexpressing or miRNA-overexpressing cells, and two-way ANOVA with Tukey's post hoc test was used to identify differences in EZH2 expression between healthy control and lupus T cells, with or without overnight stimulation. To compare the expression changes before and after EZH2 overexpression, Wilcoxon's test was performed. $P$ values less than 0.05 were considered significant.

\section{RESULTS}

Expression of EZH2, H3K27me3, and miRNA in lupus patients. We first examined the expression of EZH2 in CD4+ T cells isolated from healthy controls and lupus patients. At the protein level, when the cells were stimulated, an increase in the levels of EZH2 was observed in lupus T cells but not in healthy control T cells (Figure 1A). In addition, stimulation of the T cells led to a significant increase in EZH2 expression in lupus T cells compared to healthy control T cells. EZH2 mRNA levels were also observed to be significantly increased in CD4+ $\mathrm{T}$ cells from lupus patients compared to CD4+ $\mathrm{T}$ cells from healthy controls (Figure 1B).

Since EZH2 trimethylates the histone H3K27, we measured this histone using Western blotting. As shown in Figure 1C, there was a significant increase in H3K27me3 levels in CD4+ T cells from lupus patients compared to those from healthy controls, consistent with the observed increase in EZH2 levels in lupus samples.

Since both miR-26a and miR-101 control EZH2 expression (16), we then examined the expression of these miRNAs in healthy control and lupus naive CD4+ T cells that were stimulated overnight. The levels of both miR-26a and miR-101 were significantly reduced in lupus $\mathrm{T}$ cells compared to healthy control $\mathrm{T}$ cells (Figures 1D and $\mathrm{E}$ ). The reduction in expression of these miRNAs in lupus $\mathrm{T}$ cells is consistent with, and could potentially explain, the higher levels of EZH2 observed in lupus patients compared to healthy controls.

Control of expression of EZH2 by miR-26a and miR-101 in naive CD4+ $\mathbf{T}$ cells. To confirm the inhibitory effects of miR-26a and miR-101 on EZH2 expression in naive CD4+ $\mathrm{T}$ cells, we overexpressed either
miR-26a or miR-101 in naive CD4+ T cells isolated from healthy controls and stimulated the cells in vitro. Successful overexpression of miR-26a (Figure 2A) and

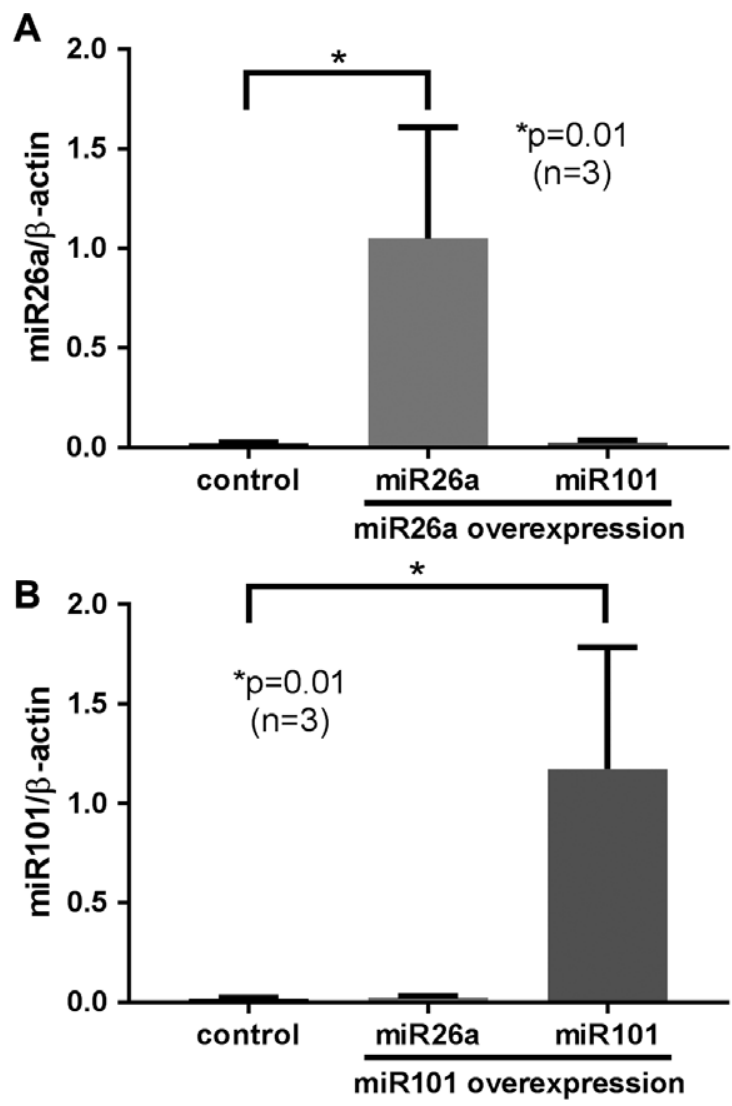

C

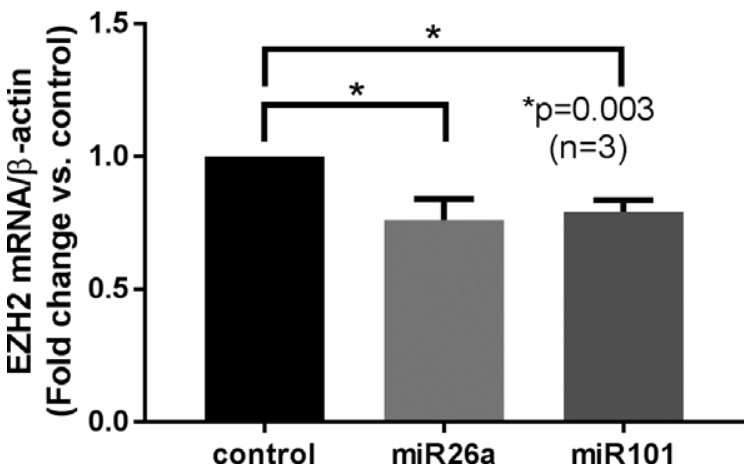

Figure 2. Effects of overexpression of microRNAs (miRNAs) miR26a and miR-101 on the levels of EZH2 mRNA in stimulated CD4+ $\mathrm{T}$ cells. $\mathbf{A}$ and $\mathbf{B}$, Naive CD4+ T cells isolated from healthy subjects were transfected with $500 \mathrm{n} M$ miR-26a, miR-101, or control miRNA and stimulated overnight. On day 3 posttransfection, overexpression of miR-26a (A) and miR101 (B) was confirmed using a TaqMan Advanced miRNA assay. C, EZH2 mRNA levels were determined in miR-26a-overexpressing and miR-101-overexpressing CD4+ T cells in comparison to control-transfected cells. Values are the mean \pm SD. 
miR-101 (Figure 2B) was first confirmed using a TaqMan Advanced miRNA assay. Indeed, both overexpression of miR-26a and overexpression of miR-101 decreased the expression of $E Z H 2$ mRNA in stimulated CD4+ Tcells (Figure 2C).

We also overexpressed both miR-26a and miR-101 simultaneously, hypothesizing that the 2 miRNAs might act synergistically to suppress EZH2 expression. Surprisingly, miR-101 appeared to negatively impact miR-26a expression, since simultaneous overexpression of both miRNAs in CD4+ Tcells led to significantly lower miR-26a levels compared to the levels in cells overexpressing miR26a alone (mean \pm SD fold increase in miR-26a levels 40.1 \pm 26.4 in miR-26a-overexpressing T cells versus $13.9 \pm 8.0$ in miR-26a and miR-101-overexpressing $\mathrm{T}$ cells $[\mathrm{n}=6$ samples per group]; $P<0.05$ ).

Effects of overexpression of $\mathrm{EZH} 2$ on genomewide DNA methylation in naive CD4+ T cells. To examine the effect of EZH2 on CD4+ T cells, EZH2 was overexpressed in naive $\mathrm{CD} 4+\mathrm{T}$ cells isolated from healthy subjects. Successful overexpression of EZH2 was confirmed at both the mRNA level (Figure 3A) and protein level (Figure 3B). Overexpression of EZH2 also led to increased H3K27me3 levels (Figure 3B).

We then evaluated DNA methylation changes in control-transfected or EZH2-overexpressing naive CD4+ $\mathrm{T}$ cells, with or without overnight stimulation. When the cells were left unstimulated, we identified 19 differentially methylated $\mathrm{CpG}$ sites in EZH2-overexpressing naive CD4+ T cells (see Supplementary Table 1, available on the Arthritis \& Rheumatology web site at http://onlinelibrary. wiley.com/doi/10.1002/art.40338/abstract), of which 8 were hypermethylated and 11 were hypomethylated in comparison to control-transfected CD4+ T cells, corresponding to 5 differentially methylated unique genes and 7 differentially methylated unique genes, respectively. When the
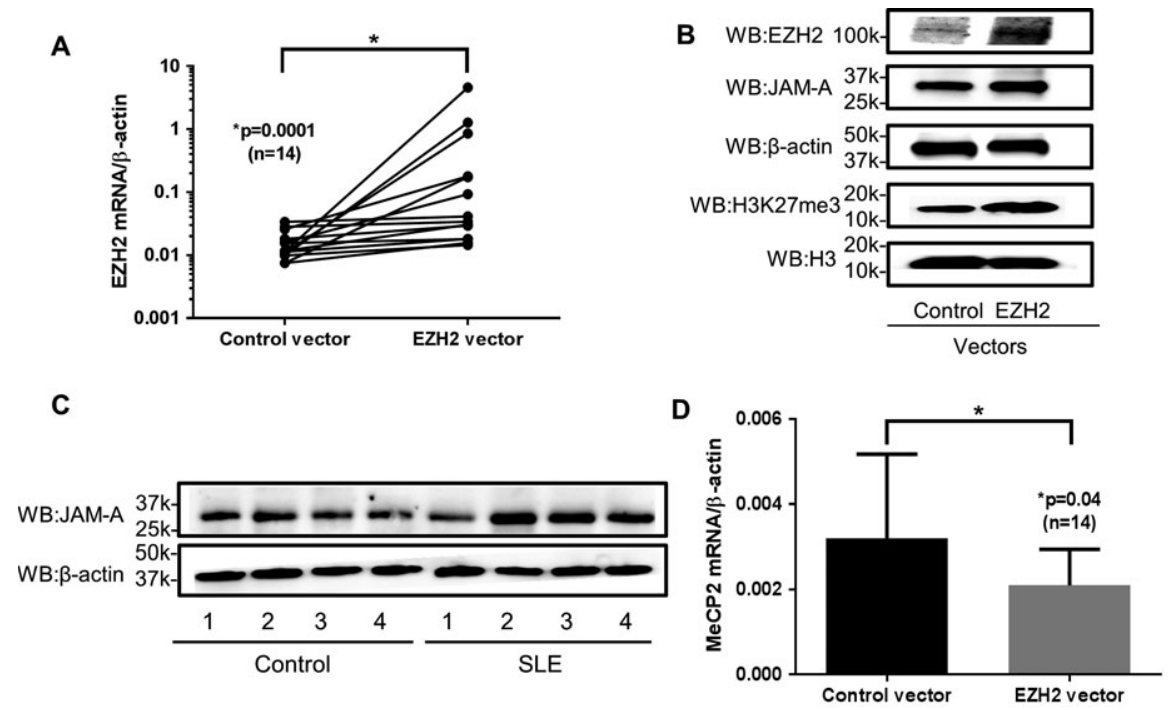

E

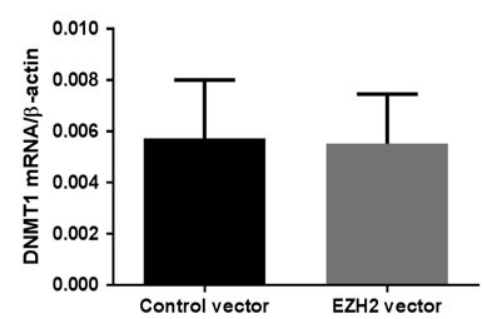

$\mathbf{F}$

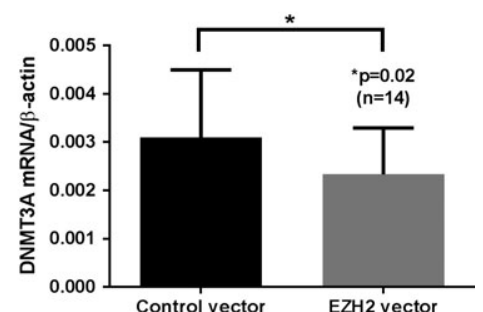

Figure 3. Effects of EZH2 overexpression in stimulated CD4+ T cells on the expression of junctional adhesion molecule A (JAM-A) and enzymes and proteins involved in DNA methylation. A, Overexpression of EZH2 mRNA in EZH2 vector-transfected CD4+ T cells, in comparison to control vector-transfected CD4+ T cells, was confirmed by quantitative polymerase chain reaction. B, Western blots (WB) (representative results from 1 of 3 experiments) show that EZH2 overexpression was paralleled by increased expression of JAM-A and trimethylated H3K27 (H3K27me3) in CD4+ T cells; $\beta$-actin and histone $\mathrm{H} 3$ were used as loading controls. C, Western blots show that JAM-A was overexpressed in stimulated naive CD4+ T cells from patients with systemic lupus erythematosus (SLE) compared to healthy controls. D-F, Expression of the methyl-CpG binding protein gene $M e C P 2$ (D) and DNA methyltransferase genes DNMT1 (E) and DNMT3A (F) was compared between EZH2-overexpressing CD4+ T cells and control cells. Results are the mean $\pm \mathrm{SD}(\mathrm{n}=14$ samples per group). 


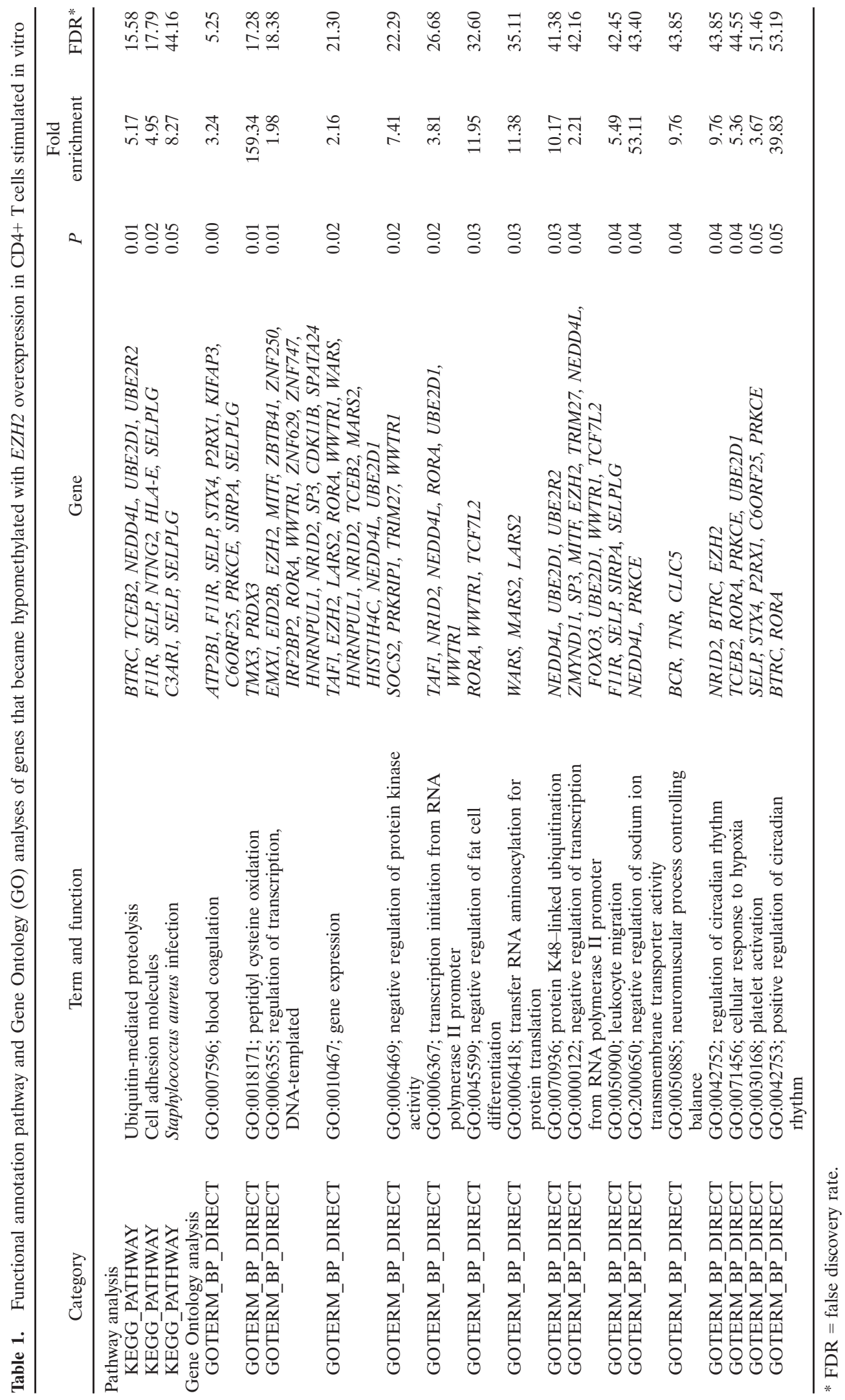


cells were stimulated overnight, a total of 324 differentially methylated $\mathrm{CpG}$ sites in EZH2-overexpressing CD4+ T cells were identified (see Supplementary Table 1). Among them, 156 were hypomethylated and 168 were hypermethylated as compared to control-transfected cells. A total of 264 differentially methylated genes were identified in EZH2-transfected CD4+ T cells following stimulation, with $\sim 50 \%$ of them being hypomethylated. The top hypomethylated gene in both unstimulated and stimulated EZH2-overexpressing CD4+ T cells was EZH2 (see Supplementary Table 1). These findings are a reflection of the overexpression of $E Z H 2$ in our experiments, since the 2 hypomethylated $\mathrm{CpG}$ sites in $E Z H 2$ are located in the gene body, suggesting that these are from the transfected $E Z H 2$ vector. In addition to EZH2, IL34 (gene for interleukin-34) was the only hypomethylated gene that was common between the unstimulated and stimulated EZH2-overexpressing CD4+ T cells.

We next used the DAVID database for gene function enrichment analysis, to group the differentially methylated genes into GO and biologic pathways. The results showing top pathways and GOs enriched among the genes that were significantly hypomethylated with EZH2 overexpression are summarized in Table 1. "Cell adhesion molecules" and "leukocyte migration" appeared to be enriched in both the functional annotation pathway and GO analyses. The genes that overlapped in both terms included F11R, SELP, and SELPLG. F11R encodes JAM-A, while $S E L P$ codes for p-selectin and SELPLG codes for $\mathrm{p}$-selectin glycoprotein ligand 1 (PSGL1). Since p-selectin is predominantly expressed on endothelial cells to mediate leukocyte adhesion through interaction with
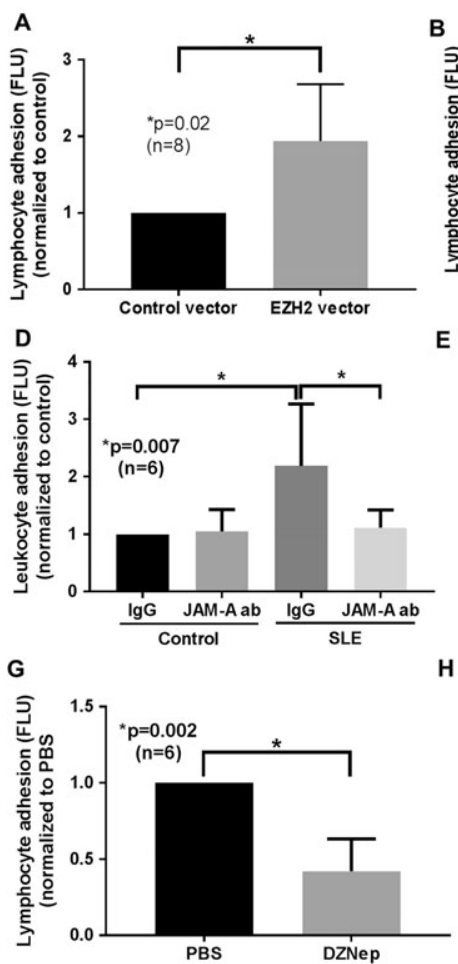

E
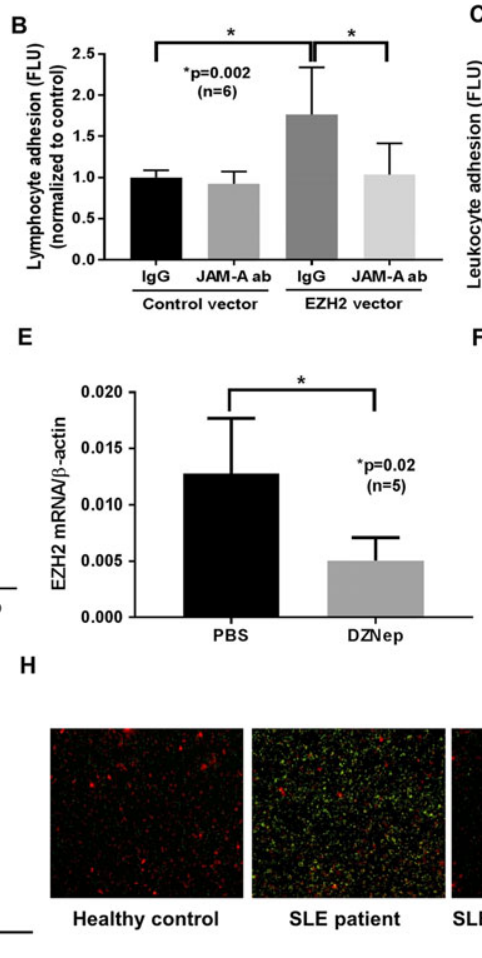

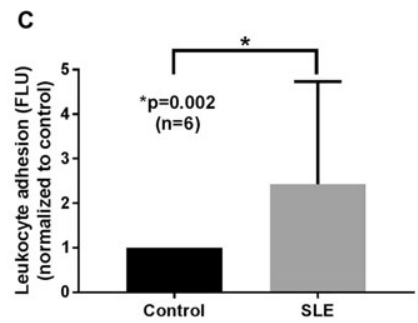

$\mathbf{F}$

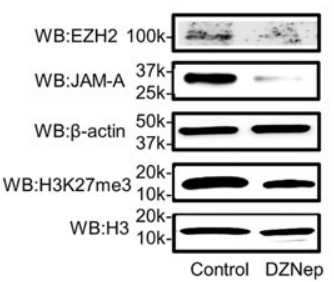

I

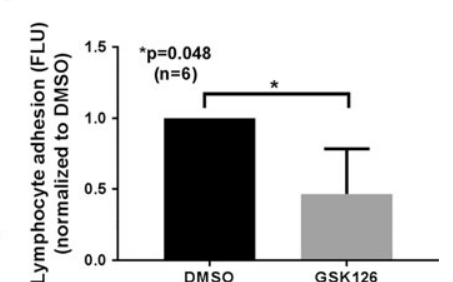

Figure 4. Effects of EZH2 on lymphocyte adhesion. A and B, Stimulated CD4+ T cells overexpressing EZH2 or transfected with a control vector were assessed for their ability to adhere to human microvascular endothelial cells (HMVECs) overall (A) and in the presence of neutralizing antibodies (ab) to junctional adhesion molecule A (JAM-A) (B). C and D, Naive CD4+ T cells from patients with systemic lupus erythematosus (SLE) and healthy controls were stimulated overnight and assessed for adhesion to HMVECs overall (C) and in the presence of anti-JAM-A (D). In these experiments, IgG was used as an isotype control. E-G, Effects of pretreatment with the EZH2 inhibitor DZNep for 3 days, using phosphate buffered saline (PBS) as a control, were assessed in stimulated CD4+ T cells isolated from lupus patients, in relation to changes in EZH2 mRNA levels (E), protein levels of EZH2, JAM-A, and trimethylated H3K27 (H3K27me3) (F), and adhesion of the cells to HMVECs (G). H, Stimulated CD4+ T cells isolated from a lupus patient (green) were assessed for their ability to adhere to HMVECs (red). Representative immunofluorescence images show adhesion assay results in cells from a healthy control subject and a lupus patient in the absence or presence of treatment with DZNep. I, Effects of pretreatment with GSK126 for 3 days, using DMSO as a control, were assessed in stimulated CD4+ T cells isolated from lupus patients, in relation to adhesion of the cells to HMVECs. Values are the mean \pm SD. Adhesion is measured as the intensity of fluorescence (FLU). WB = Western blotting. 
PSGL1 (17), we focused our subsequent studies on F11R and SELPLG in naive CD4+ T cells.

Interestingly, SELPLG was hypermethylated in unstimulated $E Z H 2$-overexpressing CD4+ Tcells, whereas it was hypomethylated after stimulation with anti-CD3 and anti-CD28 antibodies. However, the expression of PSGL1 remained unaltered with EZH2 overexpression in stimulated CD4+ T cells (data not shown), while JAM-A expression was significantly increased (Figure 3B). In addition, JAM-A was also significantly up-regulated in stimulated CD4+ T cells from lupus patients compared to those from healthy controls (Figure 3C).

Since EZH2 overexpression led to significant changes in the DNA methylome in CD4+ T cells, we examined whether EZH2 affects the expression of enzymes and proteins that are directly involved in DNA methylation. Indeed, in EZH2-overexpressing cells, the expression levels of both $\mathrm{MeCP} 2 \mathrm{mRNA}$ (Figure 3D) and DNMT3A mRNA (Figure $3 \mathrm{~F}$ ) were significantly lower than those in control-transfected $\mathrm{T}$ cells, while DNMT1 mRNA expression was not affected by EZH2 overexpression (Figure 3E).

Mediation of CD4 + $T$ cell adhesion to endothelial cells by EZH2 in patients with lupus. Since our pathway and $\mathrm{GO}$ analyses pointed to the involvement of EZH2 in leukocyte adhesion and migration (Table 1), we examined whether overexpression of $E Z H 2$ affects cell adhesion of stimulated CD4+ $\mathrm{T}$ cells to HMVECs. As shown in Figure 4A, overexpression of EZH2 increased the adhesion of T cells to HMVECs.

Since JAM-A was hypomethylated and up-regulated in EZH2-overexpressing naive CD4+ T cells (Figure $3 \mathrm{~B}$ ), we examined whether the increased adhesion capacity of these cells was mediated by JAM-A. When JAM-A was blocked with neutralizing antibodies, the increased adhesion of EZH2-overexpressing CD4+ T cells to HMVECs was normalized (Figure 4B). Since we showed that EZH2 is overexpressed in lupus naive CD4+ $\mathrm{T}$ cells following stimulation (Figures $1 \mathrm{~A}$ and $\mathrm{B}$ ), we hypothesized that these cells would behave in a manner similar to EZH2-overexpressing CD4+ T cells in terms of cell adhesion. Indeed, lupus CD4 $+\mathrm{T}$ cells showed enhanced cell adhesion to HMVECs when compared to healthy control CD4+ T cells (Figure 4C).

Since we also observed increased JAM-A expression in lupus CD4+ T cells (Figure 3C), we examined the effects of blocking the expression of JAM-A. Cell adhesion of lupus CD4+ $\mathrm{T}$ cells was significantly reduced by blockade of JAM-A (Figure 4D). These results suggest that EZH2 mediates increased $\mathrm{T}$ cell adhesion in lupus via the overexpression of JAM-A.
To determine whether inhibition of EZH2 can normalize $\mathrm{T}$ cell adhesion in lupus, we used DZNep, an EZH2 inhibitor. DZNep treatment decreased the expression of EZH2 and JAM-A, and also decreased the levels of H3K27me3 (Figures 4E and F). Furthermore, as shown in Figures $4 \mathrm{G}$ and $\mathrm{H}$, treating stimulated lupus CD4+ T cells with DZNep significantly reduced their ability to adhere to HMVECs.

To validate the effect of DZNep on T cell adhesion and determine whether this effect is dependent on H3K27me3, we used GSK126, which inhibits the enzymatic activity of EZH2, but not the expression of EZH2. Similar to what we observed in DZNep-treated cells, treatment of the cells with GSK126 led to a significant reduction in lupus $\mathrm{T}$ cell adhesion to HMVECs (Figure 4I), suggesting that EZH2-mediated T cell adhesion in lupus is dependent on the catalytic activity of EZH2.

\section{DISCUSSION}

In this study, we focused on the expression and function of EZH2 in lupus CD4+ T cells, and identified a novel role of EZH2 in T cell adhesion. We previously demonstrated that this epigenetic modulator might be mediating proinflammatory epigenetic reprogramming in naive CD4+ Tcells in lupus patients when increases in disease activity occur (10). Herein we demonstrate increased expression of EZH2 and $\mathrm{H} 3 \mathrm{~K} 27 \mathrm{me} 3$ in naive CD4+ T cells obtained from lupus patients compared to those from healthy controls following in vitro stimulation. In addition, both miR-26a and miR-101, which target EZH2, were significantly down-regulated in stimulated lupus naive CD4+ T cells. Overexpression of EZH2 in naive $\mathrm{CD} 4+\mathrm{T}$ cells from healthy individuals resulted in genome-wide DNA methylation changes, and functional enrichment analysis of the affected genes led us to examine the role of EZH2 in Tcell adhesion. We demonstrated that in vitro-stimulated CD4+ T cells, either those isolated from lupus patients or EZH2-overexpressing cells from healthy subjects, showed increased $\mathrm{T}$ cell adhesion to HMVECs as compared to their corresponding controls. This was mediated through increased expression of JAM-A in both cases. Blockade of EZH2 by DZNep and GSK126 efficiently blunted lupus $\mathrm{T}$ cell adhesion to HMVECs, suggesting that blocking EZH2 may be a therapeutic option for lupus.

As the catalytic component of a multiprotein complex, polycomb repressive complex 2, that catalyzes H3K27me3, EZH2 is involved in chromatin compaction and gene repression. When phosphorylated, it acts as a transcriptional activator instead, and this function might 
be independent of its methyltransferase activity (18). Although EZH2 preferentially targets histones, it has been shown to serve as a recruitment platform for DNA methyltransferases to directly control DNA methylation and exert their gene repression activity concurrently $(19,20)$. In this study, we showed that EZH2 overexpression led to genome-wide changes in DNA methylation. In addition to genes involved in cell adhesion and leukocyte migration, as highlighted in this study, we also discovered genes that showed differential methylation changes after $E Z H 2$ overexpression; these genes are involved in gene transcription (such as $E M X 1$ and $T A F 1$ ), ubiquitination processes (BTRC and NEDD4L), as well as immune responses (CTLA4, IL34, HLA-E, OAS2, and PGLYRP4) (Table 1; see also Supplementary Table 1, http://onlinelibrary.wiley.c om/doi/10.1002/art.40338/abstract). These findings suggest that EZH2 is involved in various cellular and physiologic processes crucial for Tcell survival and function.

The exact mechanism of how EZH2 alters the methylome in naive CD4+ T cells is not clear. However, we show that overexpression of $E Z H 2$ leads to repressed expression of both DNMT3A and MeCP2 in naive CD4+ T cells, which might result in reduced locus-specific DNA methylation or increased chromatin accessibility. In addition, the methylcytosine dioxygenase TET1 was hypermethylated in EZH2-overexpressing CD4 $+\mathrm{T}$ cells (see Supplementary Table 1). The bivalent repressive/activating effect of $E Z H 2$ could explain the similar numbers of differentially hypermethylated $\mathrm{CpG}$ sites and differentially hypomethylated $\mathrm{CpG}$ sites (168 and 156, respectively) after $E Z H 2$ overexpression in naive CD4+ T cells.

Although the function of EZH2 in Tcell differentiation remains controversial, it is clear that this pleiotropic enzyme plays a key role in maintaining T cell homeostasis $(16,21-23)$. Recently, a new function of EZH2 has been revealed. In dendritic cells and neutrophils, EZH2 was shown to control cell adhesion and migration through direct methylation of talin, which directly links integrins to the actin cytoskeleton (24). In the current study, we also showed that EZH2 mediates cell adhesion, albeit in a different cell type and with a different mechanism. Our data suggest that EZH2 promotes $\mathrm{T}$ cell adhesion to endothelial cells via, at least in part, demethylation and up-regulation of JAM-A. This was found to be true both in naive CD4+ T cells overexpressing $E Z H 2$ and in naive CD4+ T cells from lupus patients, in which expression of $\mathrm{EZH} 2$ is increased.

Despite our findings, the exact role of EZH2 in lupus $T$ cells is yet to be fully understood. The role of $\mathrm{EZH} 2$ in T cell differentiation and invasiveness is potentially crucial for lupus pathogenesis. EZH2 expression was previously examined in total CD4+ T cells from lupus patients and healthy controls (25). Those investigators showed that $E Z H 2$ mRNA levels were significantly downregulated in total CD4+ T cells from lupus patients with active disease as well as those from lupus patients with inactive disease. In contrast, we showed that EZH2 mRNA levels were elevated in naive CD4+ T cells from lupus patients compared to those from healthy controls. This discrepancy might arise from the different Tcell populations that were studied.

The process of $\mathrm{T}$ cell entry into inflamed tissue involves multiple steps, including morphologic changes occurring in T cells, adhesion of the freely circulating cells to the endothelium, and subsequent migration into the tissue. In lupus, infiltration of T cells into inflamed tissue has been well documented $(26,27)$. Increased endothelial cell activation, represented by elevated expression of E-selectin, vascular cell adhesion molecule 1, and intercellular adhesion molecule 1 , which leads to increased adhesiveness to leukocytes has been reported in the skin of lupus patients (28). In lymphocytes from lupus patients, increased levels of lymphocyte function-associated antigen 1 and very late activation antigen 4 have also been reported (29). Li and colleagues reported that lupus Tcells displayed a higher ability to adhere to hyaluronic acid-coated plates, and this was mediated by the increased phosphorylation of ezrin, radixin, and moesin and increased expression of CD44 (30). Our findings identify JAM-A as a novel molecule involved in the increased cell adhesion, and possibly tissue invasiveness, of lupus CD4+ Tcells.

JAM-A is a member of the immunoglobulin supergene family that has been identified at tight junctions between endothelial cells and epithelial cells. It is also expressed on some circulating cells, including lymphocytes (31). JAM-A is engaged in a number of binding interactions, including hemophilic interactions linking adjacent endothelial and epithelial cells to stabilize intercellular junctions, as well as heterophilic interactions with leukocyte integrins or JAMs to facilitate leukocyte influx during inflammation (32,33). The importance of JAM-A in leukocyte-endothelial cell interactions has been highlighted in studies using JAM-A-deficient mice and neutralizing antibodies to JAM-A (34-36). To our knowledge, we are the first to report that the levels of JAM-A were elevated in lupus CD4+ T cells, and to show that JAM-A mediates increased T cell adhesion to endothelial cells. In other autoimmune diseases, dysregulated expression of JAM-A has been reported (37). Decreased expression of JAM-A was observed in scleroderma skin endothelial cells, while increased expression of JAM-A was reported in scleroderma fibroblasts, which mediated myeloid cell retention on scleroderma skin (37). In peripheral blood mononuclear cells isolated from rheumatoid arthritis 
patients, F11R was hypomethylated, and an up-regulation of $F 11 R$ was observed $(38,39)$, similar to observations in the current study.

In summary, our findings indicate a novel role of EZH2 in Tcell adhesion, and a potentially pathogenic role of EZH2 overexpression in lupus. Up-regulation of EZH2 in lupus CD4+ T cells, possibly mediated by down-regulation of miR-26a and miR-101, results in an increase in H3K27me3 levels. EZH2 overexpression in T cells alters the DNA methylome and highlights the involvement of EZH2 in leukocyte adhesion and migration, via upregulation of JAM-A. We show that increased lupus CD4+ T cell adhesion is mediated by JAM-A overexpression, and that blockade of JAM-A or EZH2 can reduce the ability of CD4+ T cells to adhere to endothelial cells. These results suggest that inhibition of JAM-A or EZH2 could be a potential novel therapeutic approach in the management of lupus.

\section{ACKNOWLEDGMENT}

We thank Shaylynn Miller for her assistance in isolating naive CD4+ T cells for this study, and Dr. M. Asif Amin for his suggestions in setting up the experimental conditions for the cell adhesion assay.

\section{AUTHOR CONTRIBUTIONS}

All authors were involved in drafting the article or revising it critically for important intellectual content, and all authors approved the final version to be published. Dr. Sawalha had full access to all of the data in the study and takes responsibility for the integrity of the data and the accuracy of the data analysis.

Study conception and design. Tsou, Sawalha.

Acquisition of data. Tsou, Coit, Kilian, Sawalha.

Analysis and interpretation of data. Tsou, Coit, Sawalha.

\section{REFERENCES}

1. Wu H, Zhao M, Tan L, Lu Q. The key culprit in the pathogenesis of systemic lupus erythematosus: aberrant DNA methylation. Autoimmun Rev 2016;15:684-9.

2. Mohan C, Putterman C. Genetics and pathogenesis of systemic lupus erythematosus and lupus nephritis. Nat Rev Nephrol 2015; 11:329-41.

3. Hedrich CM, Mabert K, Rauen T, Tsokos GC. DNA methylation in systemic lupus erythematosus. Epigenomics 2017;9:505-25.

4. Coit P, Jeffries M, Altorok N, Dozmorov MG, Koelsch KA, Wren JD, et al. Genome-wide DNA methylation study suggests epigenetic accessibility and transcriptional poising of interferon-regulated genes in naive CD4+ T cells from lupus patients. J Autoimmun 2013;43:78-84.

5. Lu Q, Kaplan M, Ray D, Ray D, Zacharek S, Gutsch D, et al. Demethylation of ITGAL (CD11a) regulatory sequences in systemic lupus erythematosus. Arthritis Rheum 2002;46:1282-91.

6. Lu Q, Wu A, Tesmer L, Ray D, Yousif N, Richardson B. Demethylation of CD40LG on the inactive $\mathrm{X}$ in $\mathrm{T}$ cells from women with lupus. J Immunol 2007;179:6352-8.
7. Jeffries MA, Dozmorov M, Tang Y, Merrill JT, Wren JD, Sawalha AH. Genome-wide DNA methylation patterns in $\mathrm{CD}^{+}$ $\mathrm{T}$ cells from patients with systemic lupus erythematosus. Epigenetics 2011;6:593-601.

8. Deng C, Kaplan MJ, Yang J, Ray D, Zhang Z, McCune WJ, et al. Decreased Ras-mitogen-activated protein kinase signaling may cause DNA hypomethylation in T lymphocytes from lupus patients. Arthritis Rheum 2001;44:397-407.

9. Richardson B, Scheinbart L, Strahler J, Gross L, Hanash S, Johnson M. Evidence for impaired T cell DNA methylation in systemic lupus erythematosus and rheumatoid arthritis. Arthritis Rheum 1990;33:1665-73.

10. Coit P, Dozmorov MG, Merrill JT, McCune WJ, MaksimowiczMcKinnon K, Wren JD, et al. Epigenetic reprogramming in naive $\mathrm{CD} 4+\mathrm{T}$ cells favoring $\mathrm{T}$ cell activation and non-Th1 effector $\mathrm{T}$ cell immune response as an early event in lupus flares. Arthritis Rheumatol 2016;68:2200-9.

11. Hochberg MC. Updating the American College of Rheumatology revised criteria for the classification of systemic lupus erythematosus. Arthritis Rheum 1997;40:1725.

12. Bombardier C, Gladman DD, Urowitz MB, Caron D. Chang DH, and the Committee on Prognosis Studies in SLE. Derivation of the SLEDAI: a disease activity index for lupus patients. Arthritis Rheum 1992;35:630-40.

13. Huang da W, Sherman BT, Lempicki RA. Systematic and integrative analysis of large gene lists using DAVID bioinformatics resources. Nat Protoc 2009;4:44-57.

14. Huang da W, Sherman BT, Lempicki RA. Bioinformatics enrichment tools: paths toward the comprehensive functional analysis of large gene lists. Nucleic Acids Res 2009;37:1-13.

15. Shu Q, Amin MA, Ruth JH, Campbell PL, Koch AE. Suppression of endothelial cell activity by inhibition of TNF $\alpha$. Arthritis Res Ther 2012;14:R88.

16. Zhao E, Maj T, Kryczek I, Li W, Wu K, Zhao L, et al. Cancer mediates effector $\mathrm{T}$ cell dysfunction by targeting microRNAs and EZH2 via glycolysis restriction. Nat Immunol 2016;17:95-103.

17. Vestweber D, Blanks JE. Mechanisms that regulate the function of the selectins and their ligands. Physiol Rev 1999;79:181-213.

18. Yan J, Li B, Lin B, Lee PT, Chung TH, Tan J, et al. EZH2 phosphorylation by JAK3 mediates a switch to noncanonical function in natural killer/T-cell lymphoma. Blood 2016;128:948-58.

19. Vire E, Brenner C, Deplus R, Blanchon L, Fraga M, Didelot C, et al. The Polycomb group protein EZH2 directly controls DNA methylation. Nature 2006;439:871-4.

20. Kanduri M, Sander B, Ntoufa S, Papakonstantinou N, Sutton LA, Stamatopoulos K, et al. A key role for EZH2 in epigenetic silencing of HOX genes in mantle cell lymphoma. Epigenetics 2013;8:1280-8.

21. Yang XP, Jiang K, Hirahara K, Vahedi G, Afzali B, Sciume G, et al. EZH2 is crucial for both differentiation of regulatory T cells and T effector cell expansion. Sci Rep 2015;5:10643.

22. Jacob E, Hod-Dvorai R, Ben-Mordechai OL, Boyko Y, Avni O. Dual function of Polycomb group proteins in differentiated murine T helper $\left(\mathrm{CD}^{+}\right)$cells. J Mol Signal 2011;6:5.

23. Zhang Y, Kinkel S, Maksimovic J, Bandala-Sanchez E, Tanzer MC, Naselli G, et al. The Polycomb repressive complex 2 governs life and death of peripheral T cells. Blood 2014;124:737-49.

24. Gunawan M, Venkatesan N, Loh JT, Wong JF, Berger H, Neo $\mathrm{WH}$, et al. The methyltransferase Ezh2 controls cell adhesion and migration through direct methylation of the extranuclear regulatory protein talin. Nat Immunol 2015;16:505-16.

25. Hu N, Qiu X, Luo Y, Yuan J, Li Y, Lei W, et al. Abnormal histone modification patterns in lupus CD4+ T cells. J Rheumatol 2008;35:804-10.

26. Tsokos GC. Systemic lupus erythematosus. N Engl J Med 2011; 365:2110-21.

27. Murata H, Matsumura R, Koyama A, Sugiyama T, Sueishi M, Shibuya $\mathrm{K}$, et al. $\mathrm{T}$ cell receptor repertoire of $\mathrm{T}$ cells in the 
kidneys of patients with lupus nephritis. Arthritis Rheum 2002;46: 2141-7.

28. Belmont HM, Buyon J, Giorno R, Abramson S. Up-regulation of endothelial cell adhesion molecules characterizes disease activity in systemic lupus erythematosus: the Shwartzman phenomenon revisited. Arthritis Rheum 1994;37:376-83.

29. Takeuchi T, Amano K, Sekine H, Koide J, Abe T. Upregulated expression and function of integrin adhesive receptors in systemic lupus erythematosus patients with vasculitis. J Clin Invest 1993; 92:3008-16.

30. Li Y, Harada T, Juang YT, Kyttaris VC, Wang Y, Zidanic M et al. Phosphorylated ERM is responsible for increased $\mathrm{T}$ cell polarization, adhesion, and migration in patients with systemic lupus erythematosus. J Immunol 2007;178:1938-47.

31. Williams LA, Martin-Padura I, Dejana E, Hogg N, Simmons DL. Identification and characterisation of human junctional adhesion molecule (JAM). Mol Immunol 1999;36:1175-88.

32. Ostermann G, Weber KS, Zernecke A, Schroder A, Weber C. JAM-1 is a ligand of the $\beta$ integrin LFA- 1 involved in transendothelial migration of leukocytes. Nat Immunol 2002;3:151-8.

33. Weber C, Fraemohs L, Dejana E. The role of junctional adhesion molecules in vascular inflammation. Nat Rev Immunol 2007;7:467-77.
34. Cera MR, del Prete A, Vecchi A, Corada M, Martin-Padura I, Motoike T, et al. Increased DC trafficking to lymph nodes and contact hypersensitivity in junctional adhesion molecule-A-deficient mice. J Clin Invest 2004;114:729-38.

35. Zernecke A, Liehn EA, Fraemohs L, von Hundelshausen P,Koenen $\mathrm{RR}$, Corada $\mathrm{M}$, et al. Importance of junctional adhesion moleculeA for neointimal lesion formation and infiltration in atherosclerosis-prone mice. Arterioscler Thromb Vasc Biol 2006;26:e10-3.

36. Del Maschio A, de Luigi A, Martin-Padura I, Brockhaus M, Bartfai T, Fruscella P, et al. Leukocyte recruitment in the cerebrospinal fluid of mice with experimental meningitis is inhibited by an antibody to junctional adhesion molecule (JAM). J Exp Med 1999;190:1351-6.

37. Hou Y, Rabquer BJ, Gerber ML, del Galdo F, Jimenez SA, Haines GK III, et al. Junctional adhesion molecule-A is abnormally expressed in diffuse cutaneous systemic sclerosis skin and mediates myeloid cell adhesion. Ann Rheum Dis 2010;69:249-54.

38. Liu CC, Fang TJ, Ou TT, Wu CC, Li RN, Lin YC, et al. Global DNA methylation, DNMT1, and MBD2 in patients with rheumatoid arthritis. Immunol Lett 2011;135:96-9.

39. Fang TJ, Lin CH, Lin YZ, Li RN, Ou TT, Wu CC, et al. F11R mRNA expression and promoter polymorphisms in patients with rheumatoid arthritis. Int J Rheum Dis 2016;19:127-33. 